\title{
Trifodothyronine is Independently Associated with Metabolic Syndrome in Euthyroid Middle-aged Subjects
}

Hye Jeong Kim¹, Ji Cheol Bae², Hyeong Kyu Park', Dong Won Byun ${ }^{1}$, Kyoil Suh¹, Myung Hi Yoo', Jae Hyeon Kim², Yong-Ki Min², Sun Wook Kim², and Jae Hoon Chung²

1Division of Endocrinology and Metabolism, Department of Internal Medicine, Soonchunhyang University Hospital, Soonchunhyang University College of Medicine ${ }^{2}$ Division of Endocrinology and Metabolism, Department of Medicine, Samsung Medical Center, Sungkyunkwan University School of Medicine

\section{INTRODUCTION}

Recent studies have shown associations of thyroid hormone level $s$ with metabolic syndrome (MetS) among euthyroid individuals though there have been some inconsistencies. We evaluated the relationships between thyroid hormones and MetS components in euthyroid middle-aged subjects in a large cohort.

\section{METHODS}

Retrospective analysis of 13,496 euthyroid middle-aged subjects who participated in comprehensive health examinations was performed.

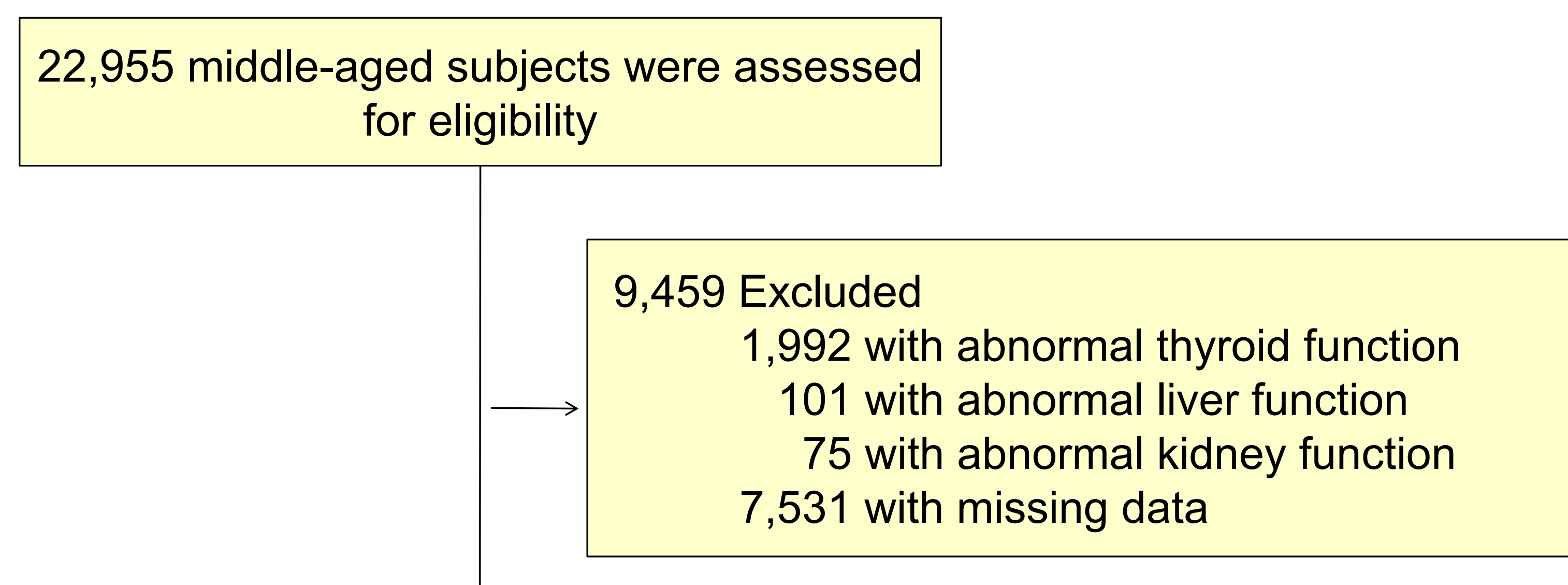

13,496 euthyroid subjects

11,832 without metabolic syndrome
1,664 with metabolic syndrome

\section{RESULTS}

Baseline characteristics of study subjects.

\begin{tabular}{|c|c|c|c|c|}
\hline \multirow[b]{2}{*}{ Variables } & \multicolumn{3}{|c|}{ Metabolic syndrome } & \multirow{2}{*}{$\begin{array}{c}\text { Overall } \\
(\mathrm{N}=13,496)\end{array}$} \\
\hline & $\begin{array}{c}\text { No } \\
(n=11,832)\end{array}$ & $\begin{array}{c}\text { Yes } \\
(n=1,664) \\
\end{array}$ & $p$ value & \\
\hline Male & $6,852(58 \%)$ & $1,316(79 \%)$ & $<0.001$ & $8,168(61 \%)$ \\
\hline Age (years) & $50.5 \pm 6.6$ & $52.3 \pm 6.5$ & $<0.001$ & $50.7 \pm 6.6$ \\
\hline $\begin{array}{l}\text { Smoking } \\
\text { (current/former/never) (\%) }\end{array}$ & $17 / 29 / 54$ & $27 / 37 / 36$ & $<0.001$ & $19 / 30 / 51$ \\
\hline BMI $\left(\mathrm{kg} / \mathrm{m}^{2}\right)$ & $23.4 \pm 2.6$ & $26.5 \pm 2.4$ & $<0.001$ & $23.8 \pm 2.7$ \\
\hline Overweight $(25 \leq \mathrm{BMI}<30)$ & $2,784(24 \%)$ & $1,258(76 \%)$ & $<0.001$ & $4,042(30 \%)$ \\
\hline Obesity (BMI $\geq 30)$ & $134(1 \%)$ & $109(7 \%)$ & $<0.001$ & $243(2 \%)$ \\
\hline Body fat $(\%)$ & $23.3 \pm 6.1$ & $25.7 \pm 5.7$ & 0.004 & $23.6 \pm 6.1$ \\
\hline Male & $20.2 \pm 4.4$ & $23.8 \pm 4.1$ & $<0.001$ & $20.8 \pm 4.5$ \\
\hline Female & $27.7 \pm 5.4$ & $33.1 \pm 4.9$ & $<0.001$ & $28.0 \pm 5.5$ \\
\hline Abdominal fat (\%) & $0.88 \pm 0.06$ & $0.93 \pm 0.06$ & $<0.001$ & $0.88 \pm 0.06$ \\
\hline Male & $0.89 \pm 0.06$ & $0.93 \pm 0.05$ & $<0.001$ & $0.90 \pm 0.06$ \\
\hline Female & $0.85 \pm 0.06$ & $0.91 \pm 0.06$ & $<0.001$ & $0.86 \pm 0.06$ \\
\hline Systolic BP (mmHg) & $109.9 \pm 14.2$ & $121.0 \pm 15.3$ & $<0.001$ & $111.3 \pm 14.8$ \\
\hline Diastolic BP (mmHg) & $67.7 \pm 9.8$ & $74.4 \pm 10.0$ & $<0.001$ & $68.5 \pm 10.0$ \\
\hline Total cholesterol (mg/dL) & $188.0 \pm 30.7$ & $191.9 \pm 33.2$ & $<0.001$ & $188.5 \pm 31.0$ \\
\hline LDL cholesterol (mg/dL) & $122.8 \pm 27.9$ & $125.4 \pm 29.9$ & 0.001 & $123.1 \pm 28.2$ \\
\hline HDL cholesterol (mg/dL) & $59.4 \pm 13.5$ & $47.3 \pm 10.9$ & $<0.001$ & $57.9 \pm 13.8$ \\
\hline Male & $55.5 \pm 11.9$ & $47.2 \pm 11.3$ & $<0.001$ & $54.2 \pm 12.2$ \\
\hline Female & $64.8 \pm 13.8$ & $47.7 \pm 9.2$ & $<0.001$ & $63.6 \pm 14.2$ \\
\hline Triglycerides (mg/dL) & $114.7 \pm 63.9$ & $214.0 \pm 102.5$ & $<0.001$ & $126.9 \pm 77.1$ \\
\hline Fasting glucose (mg/dL) & $87.5 \pm 12.9$ & $103.7 \pm 23.8$ & $<0.001$ & $89.5 \pm 15.6$ \\
\hline $\mathrm{HbA} 1 \mathrm{c}(\%)$ & $5.3 \pm 0.5$ & $5.9 \pm 0.9$ & $<0.001$ & $5.4 \pm 0.6$ \\
\hline HOMA-IR & $1.9 \pm 0.9$ & $2.9 \pm 1.5$ & $<0.001$ & $2.1 \pm 1.1$ \\
\hline TSH (mU/L) & $2.4 \pm 1.3$ & $2.3 \pm 1.3$ & 0.017 & $2.3 \pm 1.3$ \\
\hline T3 (nmol/L) & $1.72 \pm 0.28$ & $1.78 \pm 0.29$ & $<0.001$ & $1.72 \pm 0.28$ \\
\hline $\mathrm{T} 4$ (nmol/L) & $108.6 \pm 17.9$ & $108.3 \pm 18.4$ & 0.521 & $108.6 \pm 17.9$ \\
\hline T3 to T4 ratio & $0.016 \pm 0.003$ & $0.017 \pm 0.003$ & $<0.001$ & $0.016 \pm 0.003$ \\
\hline
\end{tabular}

Means for thyroid hormone levels by select metabolic syndrome components.

\begin{tabular}{|c|c|c|c|c|c|}
\hline \multirow[t]{2}{*}{ Variables } & \multirow[b]{2}{*}{$\mathrm{n}(\%)$} & \multicolumn{4}{|c|}{ Thyroid hormones } \\
\hline & & $\mathrm{TSH}(\mathrm{mU} / \mathrm{L})$ & T3 (nmol/L) & $\mathrm{T} 4(\mathrm{nmol} / \mathrm{L})$ & T3 to T4 ratio \\
\hline \multicolumn{6}{|l|}{$\mathrm{BMI}\left(\mathrm{kg} / \mathrm{m}^{2}\right)$} \\
\hline Underweight $(<18.5)$ & $219(2 \%)$ & $2.6 \pm 1.3^{\star a}$ & $1.67 \pm 0.28^{\star a}$ & $109.7 \pm 17.4 \ddagger^{\mathrm{a}}$ & $0.015 \pm 0.003^{* a}$ \\
\hline Normal (18.5-24.9) & $8,992(66 \%)$ & $2.4 \pm 1.3^{\star a}$ & $1.71 \pm 0.28^{\star b}$ & $108.8 \pm 17.9 \ddagger^{\mathrm{a}, \mathrm{b}}$ & $0.016 \pm 0.003^{\star b}$ \\
\hline Overweight (25-29.9) & $4,042(30 \%)$ & $2.2 \pm 1.2^{* b}$ & $1.75 \pm 0.28^{\star c}$ & $108.0 \pm 17.9 \ddagger^{\mathrm{a}, \mathrm{c}}$ & $0.017 \pm 0.003^{* c}$ \\
\hline Obesity $(\geq 30)$ & $243(2 \%)$ & $2.2 \pm 1.2^{* b}$ & $1.79 \pm 0.26^{\star d}$ & $110.9 \pm 20.0 \ddagger^{a, b}$ & $0.017 \pm 0.003^{* c}$ \\
\hline \multicolumn{6}{|l|}{ Systolic BP (mmHg) } \\
\hline$<130$ & $10,272(76 \%)$ & $2.3 \pm 1.3$ & $1.71 \pm 0.28^{*}$ & $108.3 \pm 17.8^{*}$ & $0.016 \pm 0.003 \dagger$ \\
\hline$\geq 130$ & $3,224(24 \%)$ & $2.3 \pm 1.3$ & $1.76 \pm 0.29^{*}$ & $109.7 \pm 18.3^{*}$ & $0.017 \pm 0.003 \dagger$ \\
\hline \multicolumn{6}{|l|}{ Diastolic BP (mmHg) } \\
\hline$<85$ & $10,756(80 \%)$ & $2.3 \pm 1.3$ & $1.71 \pm 0.28^{*}$ & $108.3 \pm 17.8^{*}$ & $0.016 \pm 0.003 \ddagger$ \\
\hline$\geq 85$ & $2,739(20 \%)$ & $2.3 \pm 1.3$ & $1.76 \pm 0.29^{*}$ & $109.9 \pm 18.5^{*}$ & $0.017 \pm 0.003 \ddagger$ \\
\hline \multicolumn{6}{|l|}{ HDL cholesterol (mg/dL) } \\
\hline low & $1,555(12 \%)$ & $2.5 \pm 1.3^{*}$ & $1.68 \pm 0.28^{*}$ & $108.8 \pm 17.7$ & $0.016 \pm 0.003^{*}$ \\
\hline high & $11,941(88 \%)$ & $2.3 \pm 1.3^{*}$ & $1.73 \pm 0.28^{*}$ & $108.6 \pm 17.9$ & $0.017 \pm 0.003^{*}$ \\
\hline \multicolumn{6}{|l|}{ Triglycerides (mg/dL) } \\
\hline$<150$ & $9,966(74 \%)$ & $2.3 \pm 1.3$ & $1.70 \pm 0.28^{*}$ & $108.8 \pm 17.9$ & $0.016 \pm 0.003^{*}$ \\
\hline$\geq 150$ & $3,530(26 \%)$ & $2.4 \pm 1.3$ & $1.80 \pm 0.28^{*}$ & $108.1 \pm 18.1$ & $0.017 \pm 0.003^{*}$ \\
\hline \multicolumn{6}{|l|}{ Fasting glucose (mg/dL) } \\
\hline$<100$ & $11,484(85 \%)$ & $2.4 \pm 1.3^{*}$ & $1.72 \pm 0.28^{*}$ & $108.6 \pm 17.8$ & $0.016 \pm 0.003^{*}$ \\
\hline$\geq 100$ & $2,012(15 \%)$ & $2.2 \pm 1.2^{*}$ & $1.75 \pm 0.29^{*}$ & $108.6 \pm 18.6$ & $0.017 \pm 0.003^{*}$ \\
\hline \multicolumn{6}{|l|}{$\mathrm{HbA} 1 \mathrm{c}(\%)$} \\
\hline$<5.7$ & $10,636(79 \%)$ & $2.4 \pm 1.3 \ddagger^{a, b}$ & $1.72 \pm 0.28^{* a}$ & $108.5 \pm 17.8 \dagger^{a}$ & $0.016 \pm 0.003^{* a}$ \\
\hline $5.7-6.4$ & $1,992(15 \%)$ & $2.3 \pm 1.3 \ddagger^{\mathrm{a}, \mathrm{b}}$ & $1.75 \pm 0.29^{* b}$ & $108.6 \pm 17.8 \dagger^{a}$ & $0.017 \pm 0.003^{* \mathrm{~b}}$ \\
\hline$\geq 6.5$ & $868(6 \%)$ & $2.2 \pm 1.3 \ddagger^{\mathrm{b}, \mathrm{c}}$ & $1.73 \pm 0.30^{* a}$ & $110.4 \pm 19.6 \dagger^{b}$ & $0.016 \pm 0.003^{* a}$ \\
\hline
\end{tabular}

BMI, body mass index; BP, blood pressure; HDL, high-density lipoprotein.

${ }^{*} p<0.001, \dagger 0.001 \leq p<0.01, \ddagger 0.01 \leq p<0.05$.

$\mathrm{a}, \mathrm{b}, \mathrm{c}, \mathrm{d}$ The same letters indicate non-significant difference between groups based on Tukey's multiple co mparison test.

The proportion of subjects with metabolic syndrome by thyroid hormone quartile categories within the euthyroid range.

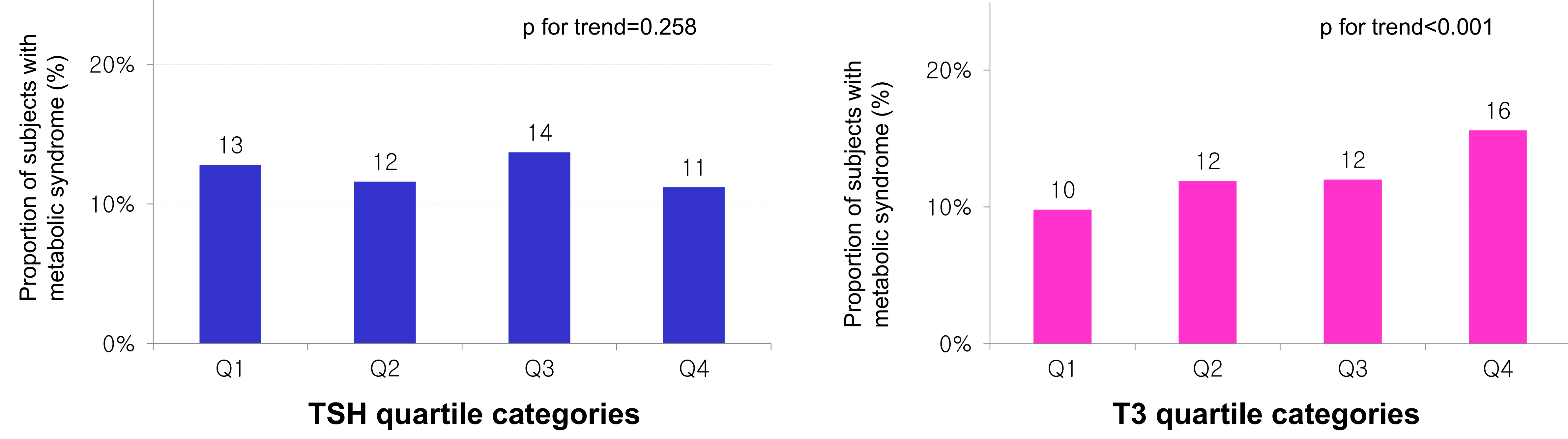

Risk of metabolic syndrome in euthyroid subjects according to hormone quartiles.

\begin{tabular}{lcccc}
\hline Thyroid hormone & Metabolic syndrome/ & \multicolumn{3}{c}{ OR $(95 \% \mathrm{Cl})$} \\
\cline { 3 - 5 } categories & Total $\mathrm{n}(\%)$ & Model 1 & Model 2 & Model 3 \\
\hline TSH quartiles & & & & \\
0.4-1.4 mU/L & $481 / 3,744(13 \%)$ & 1.000 & 1.000 & 1.000 \\
$1.5-2.1 \mathrm{mU} / \mathrm{L}$ & $395 / 3,414(12 \%)$ & $0.888(0.747-1.055)$ & $0.919(0.772-1.095)$ & $0.926(0.727-1.178)$ \\
$2.2-3.0 \mathrm{mU} / \mathrm{L}$ & $423 / 3,085(14 \%)$ & $1.078(0.909-1.279)$ & $1.170(0.983-1.391)$ & $1.245(0.982-1.577)$ \\
$3.1-5.0 \mathrm{mU} / \mathrm{L}$ & $365 / 3,253(11 \%)$ & $0.857(0.719-1.023)$ & $1.016(0.848-1.217)$ & $1.134(0.887-1.452)$ \\
T3 quartiles & & & & \\
$1.10-1.51 \mathrm{nmol} / \mathrm{L}$ & $323 / 3,307(10 \%)$ & 1.000 & 1.000 & 1.000 \\
$1.52-1.69 \mathrm{nmol} / \mathrm{L}$ & $403 / 3,373(12 \%)$ & $1.254(1.074-1.464) \dagger$ & $1.217(1.040-1.424) \ddagger$ & $1.025(0.829-1.268)$ \\
$1.70-1.89 \mathrm{nmol} / \mathrm{L}$ & $415 / 3,470(12 \%)$ & $1.255(1.076-1.464) \dagger$ & $1.169(1.001-1.366) \ddagger$ & $1.000(0.809-1.235)$ \\
$1.90-2.90 \mathrm{nmol} / \mathrm{L}$ & $523 / 3,346(16 \%)$ & $1.712(1.476-1.984)^{*}$ & $1.570(1.351-1.824)^{*}$ & $1.249(1.020-1.529) \dagger$ \\
T4 quartiles & & & & \\
$64.0-96.0 \mathrm{nmol} / \mathrm{L}$ & $481 / 3,631(13 \%)$ & 1.000 & 1.000 & 1.000 \\
$96.1-107.0 \mathrm{nmol} / \mathrm{L}$ & $390 / 3,387(12 \%)$ & $0.852(0.716-1.014)$ & $0.870(0.730-1.038)$ & $0.867(0.685-1.097)$ \\
$107.1-119.5 \mathrm{nmol} / \mathrm{L}$ & $354 / 3,153(11 \%)$ & $0.828(0.693-0.990) \dagger$ & $0.814(0.680-0.975) \dagger$ & $0.746(0.582-0.956) \dagger$ \\
$119.6-155.0 \mathrm{nmol} / \mathrm{L}$ & $439 / 3,325(13 \%)$ & $0.996(0.841-1.180)$ & $0.942(0.794-1.119)$ & $0.890(0.706-1.121)$ \\
T3 to T4 ratio quartiles & & & & \\
$0.0060-0.0140$ & $333 / 3,379(10 \%)$ & 1.000 & 1.000 & 1.000 \\
$0.0141-0.0159$ & $375 / 3,406(11 \%)$ & $1.132(0.936-1.368)$ & $1.132(0.934-1.371)$ & $1.097(0.843-1.428)$ \\
$0.0160-0.0179$ & $428 / 3,329(13 \%)$ & $1.350(1.122-1.624)^{*}$ & $1.358(1.126-1.638)^{*}$ & $1.275(0.988-1.646)$ \\
$0.0180-0.0565$ & $528 / 3,382(16 \%)$ & $1.692(1.416-2.022)^{*}$ & $1.616(1.349-1.936)^{*}$ & $1.458(1.141-1.863)^{*}$ \\
\hline
\end{tabular}

Model 1 , unadjusted; Model 2, adjusted for sex and age;

Model 3 , adjusted for sex, age, body fat percentage, smoking and HOMA-IR.

${ }^{*} \mathrm{p}<0.001, \dagger 0.001 \leq \mathrm{p}<0.01, \neq 0.01 \leq \mathrm{p}<0.05$.

\section{CONCLUSION}

Serum T3 levels are independently associated with MetS in euthyroid middle-aged subjects. 